\title{
Tangence
}

\section{Voyages en allophonie}

\section{Juan Alonso}

Numéro 59, janvier 1999

Écrivains d'ailleurs

URI : https://id.erudit.org/iderudit/025996ar

DOI : https://doi.org/10.7202/025996ar

Aller au sommaire du numéro

Éditeur(s)

Tangence

ISSN

0226-9554 (imprimé)

1710-0305 (numérique)

Découvrir la revue

Citer cet article

Alonso, J. (1999). Voyages en allophonie. Tangence, (59), 121-127.

https://doi.org/10.7202/025996ar d'utilisation que vous pouvez consulter en ligne.

https://apropos.erudit.org/fr/usagers/politique-dutilisation/ 


\section{Voyages en allophonie Juan Alonso}

Natif de Buenos Aires, j'écris maintenant dans la langue que les francophones appellent l'américain ${ }^{1}$. Le passage vers cette autre langue se fit durant mon adolescence, après que mes frères et moi avons été élevés en tant qu'Argentins typiques de manière à être reconnus comme personnages authentiques pour le reste de notre vie. J'insiste sur ce point parce qu'un changement soudain et brutal de pays, de langue et de culture est souvent perçu comme une menace pour le sens qu'a un individu de sa propre réalité. En tant qu'enfants d'immigrants en Argentine, nous avons reproduit les gestes et les sons du Porteno local, un peu comme des lionceaux apprenant à chasser. Nous grandissions dans cette autre peau avec beaucoup plus de facilité que nos parents (en supposant qu'ils aient déjà essayé de revêtir cette nouvelle peau, bien sûr). Nous étions convaincus que c'était ce qu'il y avait de mieux et de plus sage à faire. (Cela me fait penser à ce vieux film, dont plus personne ne se souvient, dans lequel on trouvait le détective confucianiste Charlie Chan et son Fiston numéro un ("Number One Son"), cet étudiant de l'Americanizing College Fraternity, qui s'adressait familièrement à lui en lui disant: "Hi, Pop!" (Salut, p'pa!). Naturellement, le grand détective traitait ce troufignon avec condescendance. Il a toujours été évident que ce n'était pas tant le vocabulaire ou l'accent qui différenciaient les deux hommes, mais leur vision du monde.)

Ma mère, Anglaise d'origine, parle très bien l'espagnol, qu'elle a appris en Espagne. En Argentine, elle demeurait la plus étrangère de nous tous, celle qui déployait le plus gros accent (comme elle devait être heureuse le jour où, installés aux États-Unis, nous devenions à notre tour les étrangers!). Elle se sentait évidemment comme un poisson dans l'eau, avec son anglais maternel et sonore, qui lui valut d'être souvent sollicitée par Borges, cet être plein de gratitude et d'anglophilie éhontée que gagnait la cécité, pour lui faire la lecture. Quant à mon père, Espagnol étranger à Buenos Aires - car il n'était pas Argentin -, il fut malgré tout un critique littéraire renommé, qui consacra, à mon avis, ses

1 En français dans le texte (Note du traducteur). 
122

meilleures pages à la poésie intensément subjective et hermétique de Neruda. Après avoir été déporté par Peron, il est mort à l'Université de Harvard, dans la meilleure situation qu'il pût occuper aux Etats-Unis dans son domaine de travail, mais très malheureux, car la langue était pour lui, depuis toujours, aussi vitale que l'oxygène. Mais il ne s'était jamais adapté à l'américain, cet autre gaz qui ne l'a jamais aidé à se maintenir en vie, loin de là. Du plus profond de mon coeur, j'ai toujours cru que mon père s'est éteint - très littérairement - avec l'échec de la traduction.

Prendre cette nouvelle langue sous ma juridiction devint donc pour moi d'une importance existentielle ultime. Et à plus forte raison lorsque je sus que j'allais, de mon propre chef, me vouer à l'écriture pour le restant de mes jours, pour le meilleur et pour le pire.

Sans être un philosophe du langage, j'appris très tôt ce que tout le monde sait: quand un Français, un Yankee ou un Argentin décrivent une seule et même scène, l'objet décrit ne sera pas perçu de la même manière par aucun d'eux. Chacun y apportera sa propre sensibilité et un souffle bien à lui. C'est bien connu.

Les expériences que connut mon père dans la région du Haut Boston se présentaient, surtout à ses propres yeux, comme celles d'un Espagnol. La langue espagnole, celle de l'Espagne, le parlait. Je me souviens d'avoir senti que les choses ne pouvaient être autrement, comme je voyais ses différents mérites, vertus et même les belles actions qu'il faisait - aussi bien que les horreurs qu'il commettait. (L'adolescent américain que j'étais se sentait évoluer dans une condition ironique, celle d'un nouveau mélange de Charlie Chan et de Number One Son, mais en les appréhendant tous deux. Parce qu'à mes yeux Charlie était quelqu'un qui vivait, ou qui tentait de vivre, en traduction, alors que son fils se plaisait surtout à exprimer sa prétendue appartenance à la masse heureuse, qui avait l'avenir pour elle. Ce faisant, il triomphait, à mon avis, du vieil homme qui, par définition, "n'était pas dans le coup.. Or, pour aucun des deux, à mon avis, la nouvelle langue n'agissait comme le filtre authentique de leur expérience ni comme le moyen de l'exprimer en accord avec leur "vitalité individuée", pas même celle de Number One Son. Et c'était pourtant ce que je poursuivais, comme écrivain et comme homme.)

Lorsque mon premier roman vit enfin le jour, bien que son accueil favorable m'enchantât, je fus surpris d'apprendre que bon 
nombre de critiques américains avaient cru que mon livre avait été traduit de l'espagnol. Peut-être, me dis-je, parce que j'avais obstinément signé : Juan Alonso. Juan, plutôt que Jack.

C'est alors que je signai les deux romans subséquents: J. M. Alonso. Ce mouvement, inspiré de J.D. Salinger, T.S. Eliot, etc., qui m'a toujours frappé par ce qu'il suppose de volonté de se faire accepter par la société, comme un tenancier de bar cherchant en 1890 une bonne propriété bourgeoise, me paraissait d'une prudence amplement suffisante. Et pourtant, il arriva encore qu'on me décrive comme un étranger, un exotique. Je fus même apparenté à des précurseurs tels Santayana, Nabokov et Borges, ce qui me fit sentir à la fois flatté et (j'en suis aujourd'hui surpris par l'ingratitude que cela implique) indigné. J'étais flatté, bien sûr, de me voir rapprocher de deux de mes héros, Nabokov et Borges, qui n'ont toujours eu que de vilaines choses à dire l'un sur l'autre. Mais j'étais aussi courroucé par ce que je percevais d'emblée comme une marginalisation. Je n'étais pas "l'un des leurs ", mais un pur étranger qui leur parlait dans sa perspective à lui. (À rebours, je peux maintenant voir que rien ne peut satisfaire l'ego, que Garcia Marquez a déjà décrit comme le "petit Argentin que nous portons tous en nous .)

J'étais déterminé (et je le suis toujours) à faire de l'américain ma propre langue, c'est-à-dire une manière authentique d'exprimer et de percevoir à la fois ma réalité - donc, une véritable manière d'être - plutôt qu'une traduction dans de nouveaux mots d'un tout déjà régurgité, tiré de telle ou telle autre situation, et où le costume seul serait différent.

Je suis absolument d'accord avec ceux qui considèrent une langue comme l'expérience historique d'une culture tout entière, comme une vitalité préservée, une vision du monde, une organisation, une épuration et même une explication de l'expérience. Une langue agit, en l'occurrence, de la même manière que la mythologie. Elle est peut-être elle-même une mythologie, mais la plus exclusivement distincte et inter-reliée, la plus complètement archivée des mythologies. En clair, c'est cela même que le fils du détective Chan tentait perfidement d'éliminer avec sa mortelle manie de dire, enthousiaste et les yeux brillants: "Salut P'pa!". C'est ainsi qu'il pouvait retourner chez lui avec toute nouvelle phrase ou tout mot à la mode, reçus de la presse, invitant l'aliéné à s'identifier à un nouveau troupeau en faisant table rase de son passé. La langue serait par contre une sorte d'esprit titulaire qui 
124

nous enseigne jusqu'à la manière de sentir. Elle n'est pas l'unique professeur, loin de là. Mais elle représente une source de sagesse collectivement raffinée, avec des mots et des phrases polis et mis au point de façon décisive à travers le temps, dans une économie remarquable, éradiquant ce que l'expérience juge faux et intégrant ce qu'elle juge vrai. Mais la langue n'est pas seulement une sorte de sagesse classique tirée de notre passé (la grande difficulté qui se pose pour les allophones est de faire du passé notre passé), elle est aussi quelque chose de continuellement mouvant, s'avançant en vue de définir sa voie. Le plus souvent, elle est probablement (et, je crois, certainement) largement supérieure et beaucoup plus profonde que la sagesse individuelle. On pourrait même dire que, d'un certain point de vue, la langue est un subconscient vivant comme un animal.

Dans le même ordre d'idée, lorsque je lus quelque part que Gershom Scholem, par une espèce de philologie mystique, aurait cherché dans les mots la présence manifeste de Dieu, je compris aussitôt le sens de sa démarche. Scholem semblait croire que le Dieu vivant communiquait avec nous par le biais de la langue et de son histoire, dans la mesure où nous apprenions à l'entendre. On est loin du prétendu positivisme des philologues de mon éducation du collège. Vraisemblable ou non, ce point de vue me paraissait à tout le moins être un moyen plus probable pour entrer en contact avec un Moi supérieur que la recherche, comme l'ont pratiquée les Hégéliens, des manifestations du Geist dans l'histoire politique occidentale.

Perdre tout contact avec une langue signifierait donc que l'on vit en exil (ce que, précisément, j'ai voulu ardemment éviter), et j'y vois là quelque chose d'équivalent à la mort chez les Grecs anciens. Car cela implique, à mon avis, la perte de tout contact avec un Moi supérieur, une véritable exclusion de l'Éden. Sans aucune sensibilité permettant l'inter-relation, nous y verrions aussi mal que si nous n'y voyions rien du tout, et nous caresserions la nouvelle langue avec des doigts engourdis, refroidis comme ceux d'un mort.

Je crois même que quelqu'un qui maîtrise aussi bien la langue anglaise que Nabokov (j'ai toujours considéré qu'il avait littéralement subjugué la langue anglaise en la soumettant à une violence proprement professorale) est profondément parlé par les autres langues européennes qu'il possède, et ce, en réaction à son expérience culturelle de l'américain. Lolita en serait le meil- 
leur exemple. Mais puisque je perçois Nabokov de cette manière, je dois avouer que j'ai cherché à dépasser cette perspective d'être parlé par la langue, de manière à me nourrir d'elle et à parvenir à m'exprimer sincèrement en parlant l'américain, en y ajoutant ce que je pouvais bien y ajouter. C'est, je crois, ce que j'avais en tête un peu plus tôt lorsque je mentionnais, de façon grandiloquente, mes espoirs d'acquérir une "vitalité individuée" des nouvelles eaux où, linguistiquement, je m'apprêtais à nager. (L'idée de la "nage" me rappelle une petite anecdote très célèbre de la littérature anglaise à propos de James Joyce et de sa fille, qu'il croyait littéralement surdouée, parce que le plus souvent, elle parlait en laissant libre cours au "flot de sa conscience" (stream of consciousness"). D'aucuns l'auraient déclarée schizophrène. Ce ne fut nul autre que C.G. Jung qui, à Paris, l'examina, et, devant Joyce, il tira cette conclusion: "Là où vous nagez, elle se noie." Ce n'est pas ce que j'entendais par "vitalité individuée".)

Incidemment, Borges confessa un jour qu'il avait toujours désiré, par-dessus tout, écrire en anglais. Il appartenait à une très ancienne famille argentine qui se considérait fièrement comme partie intégrante de la Race des Maîtres, bien qu'ayant aussi des racines espagnoles et juives. (D'où la vieille blague voulant qu'un Argentin soit la plupart du temps un Italien habillé à la française qui vous affirme, en espagnol, qu'il est un véritable Anglais.) Mais malheureusement, très malheureusement, Borges finit par croire qu'il n'était pas "à la hauteur" de l'anglais. Je présume qu'il lui manquait un peu de ce doigté nécessaire pour entrer en contact avec la langue tant admirée. Je dis cela en me basant sur une autre de mes expériences où je confrontai mon propre travail à sa traduction française. Même si j'avais passablement lu de textes en français, je sentis, au moment de commenter le travail du traducteur, que j'avais beaucoup moins accès aux nuances, que j'avais beaucoup moins d'oreille et d'intuition que je ne l'eus imaginé. Mis en présence de mon texte traduit en français, je me retrouvais aliéné, dans un terre étrangère, considérablement égaré, en dépit de l'habilité que je me connaissais pour avoir réussi plusieurs examens dans cette langue.

Connaître une langue en lui étant étranger fournit sans doute amplement de matière pour la philosophie, l'intrigue ou la logique. Mais, et cela vaut pour les traductions les plus fidèles en apparence, il n'y en a généralement pas assez pour la poésie (sous toutes ses formes, y compris humoristique), laquelle 
126

dépend étroitement des richesses idiomatiques et des secrets, conscients ou inconscients, dont dispose une langue pour exprimer autant de choses, si rapidement et d'une manière si vivante.

Curieusement, les écrits de Borges en espagnol sont sans doute tout aussi brillants que corrects, mais ils laissent à peine entrevoir la richesse idiomatique dont Lorca ou Neruda étaient capables. Voilà peut-être pourquoi les fictions dépeuplées, exsangues, éblouissantes et merveilleuses de Borges sont si faciles à traduire, car elles ont génêralement pour sujets des idées, et des variations sur le thème de la Vérité.

En raison de mon admiration pour Borges, je me souviens d'avoir été fort surpris et d'abord incrédule le jour où, à grand bruit, il exprima quel dégoût - je l'ai même une fois entendu parler de "répugnance" - lui inspiraient ses propres écrits (ce qu'il appelait, dans un anglais excellent et quasi édouardien qui imitait la sonorité profonde des halls d'un musée: "my stuff"). Je crois maintenant que cet aveu n'était ni affecté, ni fourbe, et que c'est dans l'“intellectualité " très traduisible de Borges qu'il faut en chercher l'explication.

Cet élément s'appuie, selon moi, sur un événement qui se produisit plus tard quand, à New York, Borges rencontra le chroniqueur de la droite américaine, catholique et ex-membre de la CIA, William F. Buckley. Une association certes inattendue, et, pourtant, j'incline à croire que les deux hommes avaient beaucoup plus de choses en commun qu'on ne pouvait d'emblée l'imaginer. Au terme d'une longue conversation, pendant laquelle Buckley avait aimablement démontré, à la grande surprise de Borges, une aisance prodigieuse pour l'espagnol, largement supérieure à celle qu'avait Borges pour l'anglais (qui n'était certainement pas l'américain), et déployé, avec ostentation, sa connaissance des lettres espagnoles, Borges avait lâché prise et en était venu à la conclusion, après le départ de Buckley, que son espagnol était, en définitive, "trop bon". Pour cette raison, il sonnait faux, paraissait feint (bien sûr, c'était aussi mon opinion de la prose de Buckley en américain, mais c'est une autre histoire). Depuis, je me suis toujours demandé si Borges craignait que son anglais soit "trop bon", trop "buckleyesque" pour ainsi dire, pour être convaincant ou vrai, et s'il n'était pas autre chose qu'une note pédantesque, par conséquent mortellement anti-poétique. À mon avis, la réponse est oui. Car je crois que c'est pour cette raison que Borges, un des esprits les plus pénétrés de littérature que 
je connaisse, qui disait qu'il a moins vécu qu'il n'a lu, n'appréciait pas plus, en dernier lieu, ses textes en espagnol, en particulier ses fictions livresques, pleines d'une prose professorale, qu'il estimait n'être pas suffisamment "viriles". C'était son mot à lui, qui venait de l'ancienne école et qui ne se réclamait d'aucune mode (même si ce devait être son lot, aussi incroyable que cela puisse paraître, de devenir un jour à la mode).

Après toutes ces ruminations à propos des voyages en Allophonie, un territoire le plus souvent terrible, je serai peut-être bientôt porté à considérer les horizons encore plus éloignés de la poésie sans langage. Il s'agit là de quelque chose de très intéressant, si la langue continue de perdre du terrain.

Un jour, je dînais avec un professeur iranien en génie électrique, un exilé qui se sentait condamné à vivre ses derniers couchers de soleil dans les banlieues américaines. Il n'avait aucun bagage particulier quant à la littérature occidentale (et ne s'y intéressait d'ailleurs à peu près pas). Je l'ai entendu se plaindre de perdre son aisance avec le farsi, à défaut de pouvoir l'utiliser, en même temps qu'il n'arrivait pas à atteindre à plus de flexibilité dans son anglais américain, même s'il s'agissait de la langue qu'il employait le plus, professionnellement du moins.

Il se trouva que je lui racontai qu'un jour Kenneth Tynan, journaliste du New Yorker, avait réagi aux charmes de travesti de Marlene Dietrich, qui avait coutume de porter, en androgyne, un smoking, ou de gémir d'un ton intime, en déclarant qu'elle n'était qu'un "sexe sans genre". Quelque chose dans cette histoire avait touché l'ingénieur iranien, car il me demanda immédiatement de lire ses propres textes, qu'il me présenta en l'occurrence comme étant vraisemblablement de la "poésie sans langage". Il traînait ses poèmes avec lui.

Plein de sympathie et d'appréhension, je lui promis que je les lirais.

Traduit de l'anglais par Patrick Bergeron 